\title{
Integrated Analysis of fMRI-EEG Data for the Detection of Epilepsy Disorder
}

\author{
${ }^{1}$ Savita V. Raut, ${ }^{2}$ Dinkar M.Yadav, ${ }^{3}$ Ajay N. Paithane \\ ${ }^{1}$ JSPM's Rajarshi Shahu College of Engineering, Tathawade, Pune-411033 \\ savitavraut123@gmail.com \\ ${ }^{2}$ G.H. Raisoni College of Engineering \& Management, Wagholi, Pune-412207 \\ dineshyadav8@yahoo.com \\ ${ }^{3}$ JSPM's Rajarshi Shahu College of Engineering, Tathawade, Pune-411033 \\ ajaypaithane@gmail.com
}

Received: 05th August 2020, Accepted: 22nd September 2020, Published: 31st October 2020

\begin{abstract}
In this paper, new fusion model of EEG-fMRI is proposed, where the EEG correlation is developed with functional activity of the brain imaging (fMRI) to derive features. The fMRI slices and the EEG signals are correlated using the spectral correlation of selective spectral bands and the MRI spectral density in EEG-fMRI sample. Previously the mapping approach of peak spike active of EEG signal over large spectral density of MRI sample is assumed to be processed under no-artefact scenario. Secondly a redundant time feature magnitude is not effective in deriving the epileptic diagnosis of EEG signalling. Both challenges are addressed by our methodology in which feature reduction and feature mapping of selective spectral bands of EEG and MRI spectral density is proposed taking into consideration artefacts present in EEG signal. The proposed methodologies are adopted for the detection of epilepsy disorder. Experimentations are conducted in the openly available fMRI data of six subjects and active database, ACTIVE project at TelAviv Sourasky Medical Center (TASMC) Israel and comparisons are made with existing mapping models. The comparative results and analysis demonstrate the superiority and the reliability of the proposed methodology.
\end{abstract}

\section{Keywords}

Electroencephalogram (EEG), Functional Magnetic Resonance Imaging (fMRI), Epilepsy, Signal Artefacts, BOLD Signal, Spectral Correlation

\section{Introduction}

Functional magnetic resonance imaging (fMRI) is one of the main and non-invasive method that allows the localisation of brain regions in which there is a change in the level of neuronal activity during an experimental condition. Electroencephalogram (EEG) is used in detection of various human brain disorders using signal processing analysis. This test remains today the gold standard for the diagnosis of epilepsy and the localisation of the generators of epileptic activity. EEG is having good temporal resolution in the order of milliseconds while fMRI is having good spatial resolution. Simultaneous fMRI and EEG is a powerful multimodal technique for the identification of the epileptic brain networks through the correlation of Blood Oxygen Level Dependent (BOLD) signals with electrical discharges. New systems have evolved in the area of analysis and diagnosis for medical data measurement in an automated manner. In the analysis of brain activity, fMRI or EEG signal processing were used [15][28]. The processing was automated with various different types of signal conditioning tools and decision algorithms.

In the neuronal disorder, epilepsy is one of the major neuronal disease. For the diagnosis of epilepsy, EEG analysis was developed in the past [1] [4]. EEG provides a measurement of the electrical activity in the brain as a function of time by the means of electrodes placed on the scalp. Because of high temporal resolution and low costs, EEG has been found to be effective in many neuro-physiological applications related to brain-computer interaction. Various applications such as neurological disease analysis [2][3] and sensor motor restoring [4] are few examples of this application. However, as part of data analysis of these applications, it is important to maintain large input feature space as a result of this high-dimensional data, most machine learning algorithms produces inefficiency and lower processing accuracy [6]. Practically, feature separation from EEG recordings is a special way of reducing feature data. It helps to represent the condition of brain, can identify and translate effective pattern and mental state. A number of EEG features have been extracted to achieve desired goal for machine learning classifiers [16][20].

Linear extraction methods are broad, which are more applicable to stationary signal processing such as linear Fourierbased spectral analysis, auto-regressive models, time-frequency distributions. Since EEG signals are non-stationary and present evident dynamic characteristics, more popular methods such as wavelet decomposition [8] and empirical mode decomposition [9] are used. Non-stationary and non-linear representation of EEG data [30], entropic complexity 
criteria are typically offered for high classification accuracy, but increasing computational factor [10] with complex compatibility in multi-channel setups.

In processing of large EEG data two methods are used defined by [7], (i) Selection of channel corresponds to the selection of subset of electrodes contribute best performance. By selecting less number of channels, the time complexity of feature extraction and the pattern size for feature classification are greatly reduced [8]. (ii) In dimensionality reduction original feature set is reduced to smaller feature set in order to reduce the overwhelming number of extracted features [3].There is a growing interest in minimizing together the number of channels and features to be handled by the classification algorithms [11][16]. Previous approaches such as the Principal component Analysis, [17], Discriminate and Common Spatial Patterns [18] and Spatio-Spectral Decomposition [12] are popular choices for representation of EEG signal (channel or features). In order to improve computational efficiency of seizure detection, leading to a faster run time and lower power consumption. However, they experienced shortcomings, complex variations regarding non linearity and sparcity of the variations which are difficult to separate from EEG signals [19][29].

Furthermore, based on the process provided to a linear regression model, a Linear Dimensional Reduction method is chosen to select the least and most relevant features, which is not a better option in linear classifier applications [19]. Locally non-linear mapping or locally-linear information can be carefully retained by introducing discriminatory structure of the pair and attempting to divide more complex high-level data into separate group of features [20]. In dimensionality reduction approach kernel based methods are effectively been used with the properties given as [13], 1. The kernel approach is used for mapping higher dimensional data representation from a non-linear format to a linear format. 2. It reduces the complexity of diagnostic data, minimizing computational overhead by reducing parameter monitoring domain from specific feature space transformed into kernel space.

\section{Problem Statement}

The prime issue in the analysis of EEG signals for the detection of epilepsy is the artefacts observed at the measurement or the pattern variant in the measured signal due to various brain activities in the records. The automation system needs to be optimized with the advanced analysis tool to optimize these measurements to attain robustness to the distortions. Another important issue is the selection of channels corresponds to the selection of subset of electrodes, in order to avoid the redundancy of non-focal / unwanted channels; this will contribute the best performance. The complexity of EEG based epileptic seizure detection is less when numbers of required channels are low. To the best of our knowledge, mapping between EEG and fMRI is not performed with channel selection of EEG for epileptic seizure detection.

This paper introduces empirical mode decomposition to perform the promising feature selection method to get features both at low and high-frequency resolutions.

\section{Our Contributions}

In this paper, a new spectral integrated coding based on empirical mode decomposition of frequency components in EEG signal is presented. A new approach of feature reduction and feature mapping of selective spectral bands of EEG and MRI spectral density is proposed taking into consideration, artefacts present in EEG signal for the detection of epilepsy disorder. A time series analysis is proposed to derive the feature relation rather to discrete monitoring.

\section{Article Outline}

Information about EEG and fMRI is described in EEG-fMRI analysis section. The proposed methodology is discussed in Integrated fMRI Analysis (IFA) section. The experimental procedure and performance analysis section described the data and experimental procedure and investigate the performance of proposed methodology. Conclusion section concludes the paper.

\section{EEG-FMRI Analysis}

fMRI is a four dimensional brain scan ( $x, y, z$ by time), measuring relative changes in oxygenated (diamagnetic) to deoxygenated (paramagnetic) haemoglobin. As neuronal firing increases, more oxygen rich blood flows to the active brain region, generating the Blood Oxygen Level Dependent (BOLD) signal. This is an indirect measure of neural activity and neuronal firing is coupled to the BOLD signal by what is known as the Hemodynamic Response Function (HRF) and the speed of this reaction is limited by the vassal properties of blood flow. Another complication with this measure of neural activity is that the relationship between the exact location of oxygenation and the origin of neural electrical activity is uncertain [7]. The location of oxygenation cannot be assumed to be the exact origin of neural activity. In the human brain cortex (outer layer), there are large number of neurons making synchronous stimuli and producing certain rhythmic behaviours. Potential changes in the brain cortex can be obtained with a pair of electrodes placed in the skull. These potential changes consist of electrical rhythms and instant discharges. The electrical activity of the human brain can be measured and recorded with the help of EEG. The electrical activity of neurons is called as "brain waves". Every person have individual and unique brain waves. In addition, a healthy person and a person with a neurological disorder have different brain waves. When seizures occur in epileptic patient, the usual signal flow in 
the brain is distorted and consequently the structure of EEG signals changes. In general, peak-to-peak amplitude of EEG signal varies around 1-100 $\mu \mathrm{V}$ and frequency varies between $0.5-100 \mathrm{~Hz}$. EEG signals are examined in five different frequency bands. Figure 1 shows an approximate representation of EEG signals in different frequency bands. The frequency bands of EEG signals are briefly described as follows:

- Delta Band (0.1 Hz-4Hz): It is seen in deep sleep, in infancy and in patients with congenital severe brain disorders.

- Theta Band (4 Hz-8Hz): It is seen in transient parts of sleep, in awake children and in adults with emotional stress.

- Alpha Band (8Hz-13Hz): It is seen in awake but resting people.

- Beta Band (13Hz-30Hz): It is seen in people who are working and active.

- Gamma Band (30Hz and above): It is seen when the person having increased attention or related to the decision making mode of the brain.

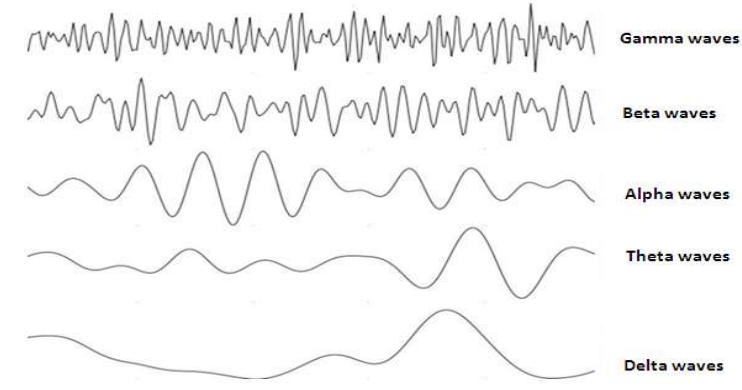

(a)

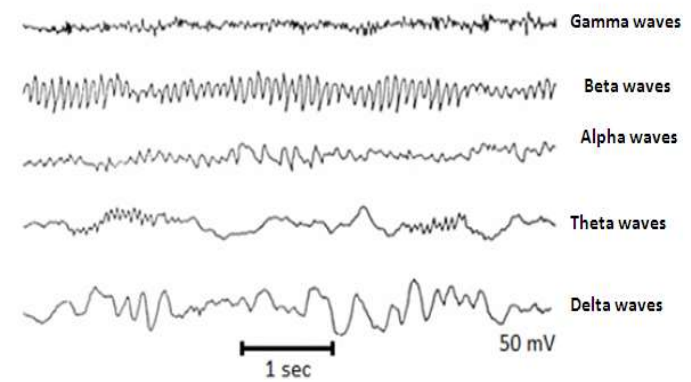

(b)

Figure 1: EEG Frequency Bands [11], (a) Normal (b) Effected

Figure 1 shows an example of an EEG signal for normal and epileptic subject. It is clear that there is a difference between seizure and non-seizure intervals. As we are able to differentiate between these intervals visually, time domain detection and prediction methods attempt to differentiate between them automatically, and evaluate the performance using different metrics such as the sensitivity, specificity, accuracy, and false-positive value.

The subject underwent simultaneous EEG-fMRI recording on a 3.0T MRI scanner with compatible scalp electrodes placed. Suitable sites for electrodes placement are frontal, parietal, temporal or occipital brain lobes. The EEG-fMRI recordings were performed in 20 min sessions of scanning; 3-5 such sessions were recorded during each patient scan. Patients were instructed to lie still and remain at rest.

The real data set used in this paper was originally collected by Keller et al. [25] and Tomer Gazit [22] under systematic experimental setup.

In the fusion model of fMRI-EEG analysis the EEG correlation is developed with the functional activity of the brain imaging to derive features.

\section{Integrated FMRI Analysis (IFA)}

The constraint of mapping features in EEG-fMRI analysis is focused to minimize;

1) The redundant spectral mapping.

2) The discrete pattern mapping of features.

The developed system model for an EEG-fMRI analysis is shown in figure 2.

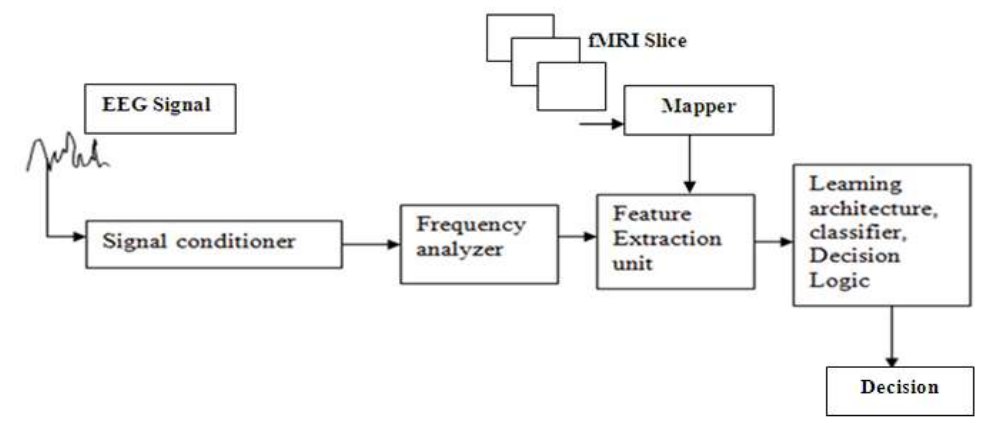

Figure 2: System Model for EEG-fMRI Integrated Coding 
The developed system has signal conditioner, frequency analyzer, feature extraction unit, learning architecture, classifier and decision logic unit. The signal conditioner pre-processes the input EEG signal, the frequency analyzer analyzes the frequency at which the signal is recorded and also check whether the frequency of given EEG signal is at normal frequency or not. Further, the EEG signal is processed to feature extraction unit. The proposed approach applies DWT for frequency extraction [11]. The obtained wavelet features are given to learning architecture to create a data base. During testing, the obtained wavelet features are given to classifier. The classifier having two inputs, one from learning architecture and another from feature extraction unit. Finally, the decision logic unit decide the given testing sample is epileptic or normal.

\section{A. Signal processing}

EEG signals with weak and low amplitude suffer from complex low frequency noise such as system interference. Thus, for the epileptic seizure analysis and detection, the EEG signal pre-processing with noise removal is especially regarded as a significant step. Due to the presence of external noise, the characteristics of signal will change, intern affects the diagnosis accuracy. For example, the range of RR-interval is $120 \mathrm{~ms}$ to $200 \mathrm{~ms}$, for an EEG signal with RRinterval range beyond these limits reflects to the wrong diagnosis. Hence, there is a need to remove external noise to increase the diagnosis accuracy. This approach proposed a non-stationary filter called as Integrated Spectral Coding (ISC) to remove the external noise accumulated in the EEG. The ISC is a non-stationary in nature. Initially, the ISC decomposes a signal into spectral bands (SB). An SB is defined as a function that satisfies the following requirements:

1. In the whole data set, the number of extrema and the number of zero-crossings must either be equal or differ at most by one.

2. At any point, the mean value of the envelope defined by the local maxima and the envelope defined by the local minima is zero.

The procedure of extracting an SB is called sifting. The sifting process is as follows:

1. Identify all the local extrema in the test data.

2. Connect all the local maxima by a cubic spline line as the upper envelope.

3. Repeat the procedure for the local minima to produce the lower envelope.

The upper and lower envelopes should cover all the data between them. Their mean is $\mathrm{m}_{1}$. The difference between the data and $\mathrm{m}_{1}$ is the first component $\mathrm{h}_{1}$ :

$h_{1}=X(t)-m_{1}$

After the first round of sifting, a crest may become a local maximum. New extrema generated in this way actually reveal the proper modes lost in the initial examination. In the subsequent sifting process, $\mathrm{h}_{1}$ can only be treated as a proto-SB. In the next step, it is treated as the data, then

$\mathrm{h}_{1}-\mathrm{m}_{11}=\mathrm{h}_{11}$

After repeated sifting up to $\mathrm{k}$ times, $\mathrm{h} 1$ becomes an $\mathrm{SB}$, that is

$\mathrm{h}_{1(\mathrm{k}-1)}-\mathrm{m}_{1 \mathrm{k}}=\mathrm{h}_{1 \mathrm{k}}$

Then, it is designated as the first SB component from the data:

$\mathrm{c}_{1}=\mathrm{h}_{1 \mathrm{k}}$

At the end of the decomposition, the data $\mathrm{s}(\mathrm{t})$ will be represented as a sum of $\mathrm{n}$ SB signals plus a residue signal, $\mathrm{s}(\mathrm{t})=\sum_{\mathrm{i}=1}^{\mathrm{n}} \mathrm{c}_{\mathrm{i}}(\mathrm{t})+\mathrm{r}_{\mathrm{n}}(\mathrm{t})$

The finally obtained signal $\mathrm{s}(\mathrm{t})$ represents the partially reconstructed signal. It is reconstructed by summing the obtained SBs with the residue signal according to eq.(5).

An ISC representation of a given query sample is illustrated in figure $3 .(\mathrm{p} 1, \mathrm{k} 1),(\mathrm{p} 2, \mathrm{k} 2), \ldots \ldots(\mathrm{pn}, \mathrm{kn})$ are the extracted feature values used as feature descriptor.

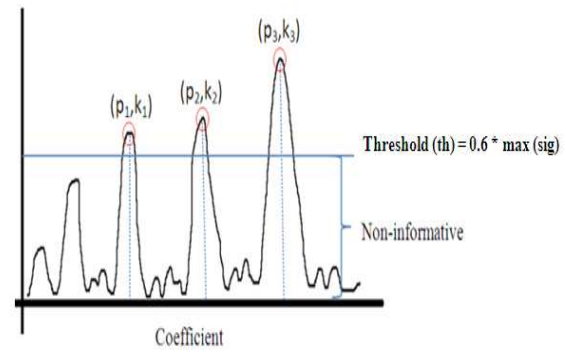

Figure 3: ISC Plot and Process of Thresholding

However, in such a coding process, information's below the threshold is totally neglected. This elimination is made based on the assumption that, only dominant edges exist for longer duration of smoothing and all the lower values are 
neglected treating as noise. For example, for a given ISC bands for a query sample, the region below the threshold is considered to be non-informative and totally neglected. This consideration leads to following observations;

1) Under semantic features having similar region representation, a false classification will appear.

2) Information's at lower regions also reveals information of signals having shorter projections such as spines.

3) Direct elimination of the entire coefficient leads to information loss as well, a random pickup will leads to higher noise density.

These problems are to be overcome to achieve higher level of retrieval accuracy in spatial semantic samples, or with sample having finer variation regions. To achieve the objective of efficient retrieval in semantic observations, a selective normalized coefficient coding is proposed.

It could be observed that the obtained ISC plot represents the region variations over different band scaling. This representation appears as a 1-D signal with random variations. Taking this observation in consideration, a normalized coefficient coding for feature representation is proposed. For the process of normalization, the ISC bands are taken as a 1-D signal and a linear wavelet decomposition using the approach of 1-D DWT coding is used. In the process of 1$\mathrm{D}$ signal decomposition, banks of recursive filters are used to decompose the signal into finer bands.

In the proposed approach of normalized coefficient coding (NCC), for the obtained ISC, a 1-D signal representation $\mathrm{x}(\mathrm{t})$, is taken as a variant of time-magnitude representation as shown in figure 4 .

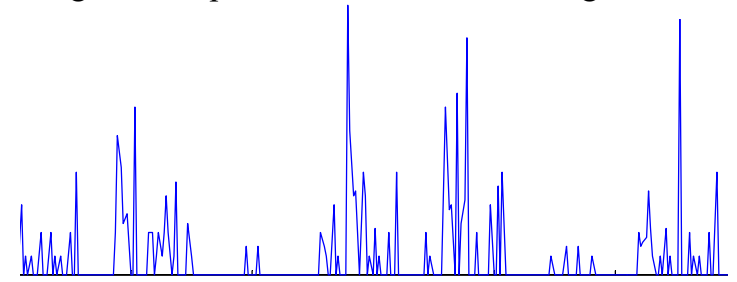

Figure 4: 1-D representation of an ISC Plot

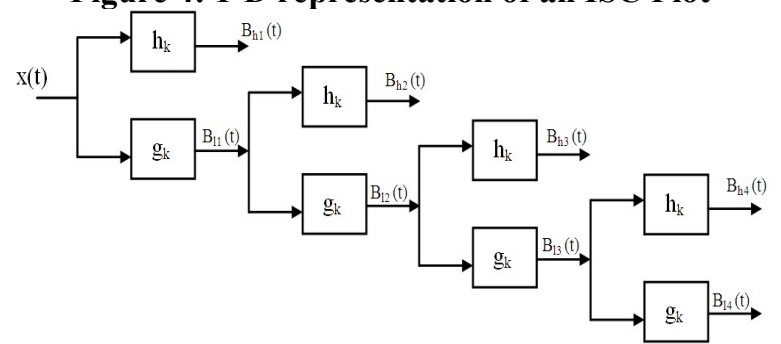

Figure 5: 1-D Wavelet Decomposition for the ISC Plot in 4-scale Level

To this 1-D signal $\mathrm{x}(\mathrm{t})$, a linear wavelet transformation is applied, to derive a bands of variant frequency contents. To perform wavelet decomposition, 'db4' and 'morlet' wavelet transformation is carried out, upto 4-scale levels. The 1$\mathrm{D}$ signal $\mathrm{x}(\mathrm{t})$ is decomposed into components belonging to different frequency channels using a collection of filters defined by db4 coefficients, referred to as sub band filtering or sub band decomposition. In this process a two-channel sub band filtering is applied with a pair one low pass $\left(g_{k}\right)$ and one high pass $\left(h_{k}\right)$ filter, to bifurcate the signal $x(t)$ into two sets of observations. An illustration to the band decomposition process is shown in figure 5.

The obtained bands $\left\{\mathrm{B}_{\mathrm{h} 1}-\mathrm{B}_{\mathrm{h} 4}\right\}$ are the decomposed detail bands revelling different frequency content at each level. $\left\{\mathrm{B}_{11}-\mathrm{B}_{14}\right\}$ are the low pass filter bands, which are decomposed in each successive band to obtain finer frequency information. Each obtained high pass filter band, reveals a finer frequency content and based on the density of these frequency contents, a decision of feature selection is made. This approach of feature selection, results in selection of feature details at lower frequency resolutions also, which were discarded in the conventional ISC approach. To derive the spectral density of these obtained bands, power spectral densities (PSD) to the obtained bands are computed. PSD is defined as a density operator which defines the variation of power over different content frequencies, in a given signal $\mathrm{x}(\mathrm{t})$.

The power spectral density (PSD) for a given signal $\mathrm{x}(\mathrm{t})$ is defined as,

$P S D, P=\lim _{T \rightarrow \infty} \frac{1}{2 T} \int_{-T}^{T} x(t)^{2} d t$

Taking each band ' $\mathrm{B}_{\mathrm{hi}}$ ' as reference, a PSD for each band, ' $\mathrm{PB}_{\mathrm{i}}$ ' is computed. The PSD features for the 4 obtained bands are then defined by,

$\mathrm{PB}_{\mathrm{i}}=\operatorname{PSD}\left(\mathrm{B}_{\mathrm{hi}}\right)$, for $\mathrm{i}=1$ to 4 .

The Band PSD's are derived as,

$P B_{i}=\lim _{T \rightarrow \infty} \frac{1}{2 T} \int_{-T}^{T} B_{h i}(t)^{2} d t$ 
From these obtained energy values, bands are selected based on a defined selection criterion, as outlined, For the obtained $\mathrm{PB}_{\mathrm{i}}$, maximum $\mathrm{PB}$ is computed, defined by,

$\mathrm{MPB}_{\mathrm{i}}=\max \left(\mathrm{PB}_{\mathrm{i}}\right)$

For $i=1$ to 4

sel_spec $_{i}=B_{i}$

$$
\text { if }\left(P B_{i} \geq\left(M P B_{i} / 2\right)\right)
$$

end

For these selected bands, 'Sel_speci' features are then computed by the approach of peak picking, as carried out in ISC approach. For each select band a maximum value is computed and all the coordinates above $60 \%$ of the peak value are taken as signal information. These approaches hence derive more informative feature information than ISC. To evaluate the developed approach a simulation model of the proposed approach is developed. These selected peaks are then processed for mapping with fMRI samples having high spectral density regions. The mapping approach is defined as a similarity function defined by $(10)$,

$s(t)=\frac{\mid\left(\sum_{k} m_{r e f}(k)-\overline{\left.m_{e f}\right)}(m(k, t)-\mid \overline{m(t))}\right.}{\sqrt{\left(\sum_{k} m_{r e f}(k)-\overline{m_{r e f}}\right)^{2}} \sqrt{\left(\sum_{k} m(k, t)-\overline{m(t)}\right)^{2}}}$

In fMRI samples spectral map coding, the active feature model is used as per the evolution of energy on a plane geometry, which is defined as a map of points. These map points are evolved towards a concentric region, where the concentric region is forced to tend towards the bounding region governed by the gradient of the evolution characteristic. The spectral map function defined by (10) defines two transitional regions, where the evolution of the feature area defined by the initial region of pixel values greater than a limiting value of $c_{1}$. The approach of evolution of feature based on the limiting condition is called as the soft thresholding approach. The second factor tries to converge towards the outer boundary region in the image. Here the region movement is based on the gradient map and the convergence of region is limited by the minimization criterion of $\vartheta(x, y, t)=0$. Here the evolution is a derived as a map of feature and as observed based on image intensities. It is observed that, the region convergence depends on the iterations given or the minimization criterion map. The convergence is achieved once the energy tends to the minimization of $\vartheta(x, y, t)$. However, the bounding condition of the energy evolution is limited with user defined values, which tends to the constraint of over computation or under computation issue. These issues lead to delay or lower accuracy in mapping process.

As the energy evolution is a function of image gradient $|\nabla \vartheta|$, the bounding condition cannot be limited for mapping. The gradient variation is observed to be dynamic for each sample and hence dynamic limiting values are appropriate. This leads to the development of automated bounding consist in spectral map coding for faster and accurate converges. To achieve this objective an approach called "convergence point" estimation is proposed. The approach is based on the characteristic analysis of the spectral map variation in fMRI sample. In the evolution of spectral map function it is observed that, once the convergence criterion met, i.e. the cost function is achieve the energy evolution is to be terminated.

The map function is defined as

$s(t)=\min \left(\left|m(x, y)-c_{i}\right|^{2}\right), \rightarrow(\vartheta(x, y, t)=0$

The convergence of mapping is defined as the minimum difference between the image and signal mapping feature. The difference vector $\mathrm{C}$ is defined as,

$C=\vartheta(t)-\vartheta(t+1)$

This is defined as the variation in the two successive observations at ' $t$ ' and ' $t+1^{\prime}$ in the spectral map function defined as energy evolution.

The termination condition is defined as,

if $C<0$,

$S=1$

else

$S=0$

end

The terminating status $\mathrm{S}$ is map high to stop the energy evolution condition once the zero cross value is obtained. The selected feature bands and the corresponding spectral bands converging with the MRI spectral variation are mapped. The mapping convergence is illustrated in figure 6 , 


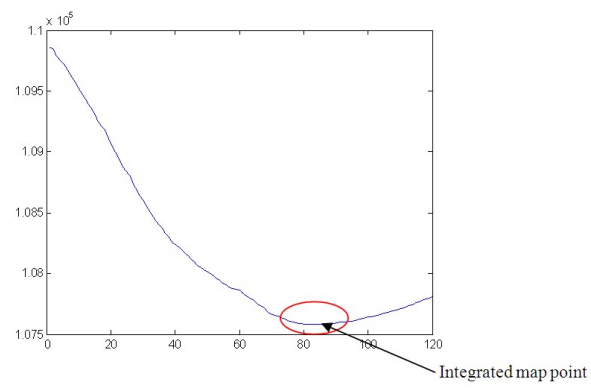

Figure 6: Integrated Map Point Convergence

This characteristic analysis illustrates a sharp variation in the spectral map evolution, where it is observed that the spectral map value tends to lower until the bounding curve regions are detected.

The operation of the developed approach is carried out in two operation stages, training and testing. Where in training process set of recorded signals are processed in a sequence and the process of feature extraction is performed. For each of the training signal, the obtained features are buffered into an array termed as data base. To train the database the features extracted from the decomposed bands are, alpha, beta, gamma and delta bands. During the process of querying the same process is repeated for the test sample and the obtained query feature is passed to a classifier to retrieve information's from the knowledge data base. For the process of classification, support vector machine (SVM) classifier is used. The classifier is designed with a Euclidian distance based approach to obtain the best set of matches from the knowledge data base [22][25]. The decision ' $\mathrm{D}$ ' for the retrieval is derived as the minimum value of the Euclidian distance defined as,

$D=\min \left(E d_{i}\right)$

Where,

Euclidian Distance, $E d_{i}=\sqrt{\sum_{i=1}^{n} Q-} d b f_{i}$

Where, $\mathrm{Q}$ is the query feature and,

$\mathrm{dbf}_{\mathrm{i}}$ is the features trained in the data base.

\section{Experimental Procedure and Performance Analysis}

To validate the proposed work, the suggested work is developed on MatlabR2015a. The proposed work is tested on a set of samples taken from StarPlus database [25] and active database [22]. The active database contains two types of recordings collected as part of the ACTIVE project at TelAviv Sourasky Medical Center (TASMC) Israel. The first set is FMRI dataset including language FMRI testing and anatomical MRI that were part of a machine learning language lateralization project. The second set is EEG-fMRI recording for the detection of the epileptic network. This dataset includes both EEG and fMRI recordings, recorded simultaneously and anatomical MRI. These samples are passed to the processing algorithm for training, where each signal is read in a sequence and the computed features are buffered in an array. This buffered information is taken as the knowledge information for classification. The process of proposed approach is carried out for a selected test sample. The processing results obtained are illustrated below.

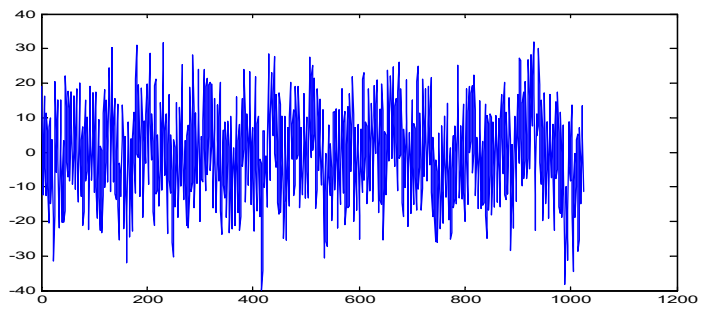

Figure 7: Test Sample

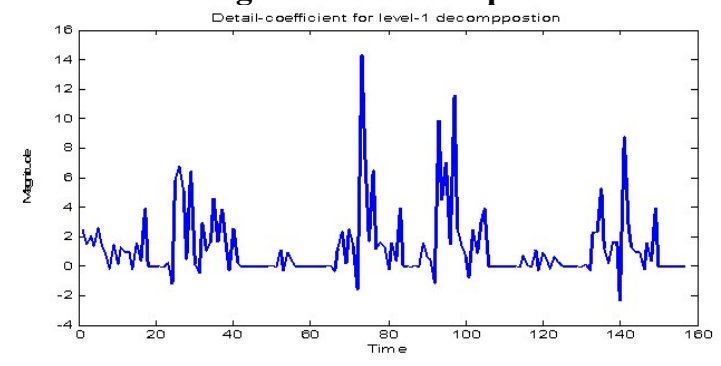

Figure 8: Detail Spectral Band at Level-1 Decomposition 
For the evaluation of developed work, a test query EEG signal is passed. An EEG signal sample is shown in figure 7. The test sample is tested for the extraction of similar train case sample from the database.

To derive the spectral band for the given test sample, the obtained coefficient information's are buffered into a linear array and the coefficients are passed to perform spectral decomposition to compute multi-spectral band decomposition. The detail bands for the given EEG signal are outlined in figures below.

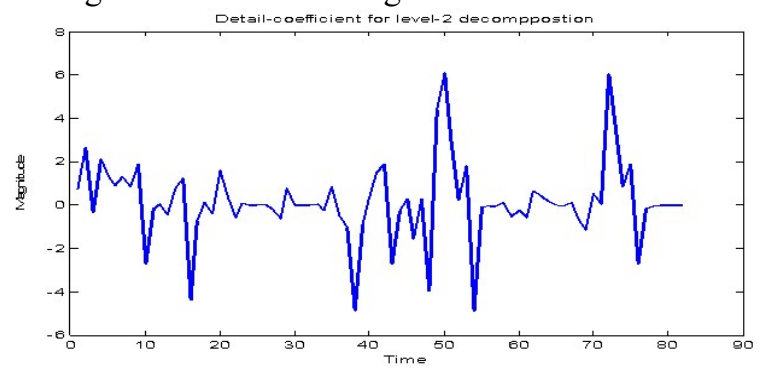

Figure 9: Detail Spectral Band at Level-2 Decomposition

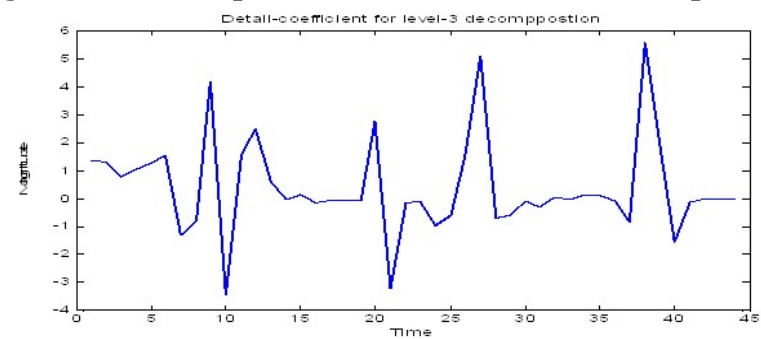

Figure 10: Detail Spectral Band at Level-3 Decomposition

A spectral decomposition is carried out using db4 wavelet transformation to get denoised EEG signal. The 3 detail bands obtained are shown in figure 8-10 respectively. It is observed that band 1 and band 3 exhibits higher coefficients variation than the other two bands, hence more coefficient information are presented in these two bands. To select the required bands for feature extraction, a spectral density using power spectral density is used. The energy density for each band is shown in figure 11

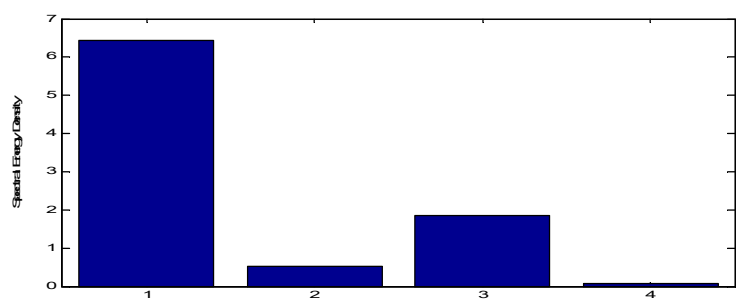

Figure 11: Spectral Energy Density for 4-Decomposed Bands

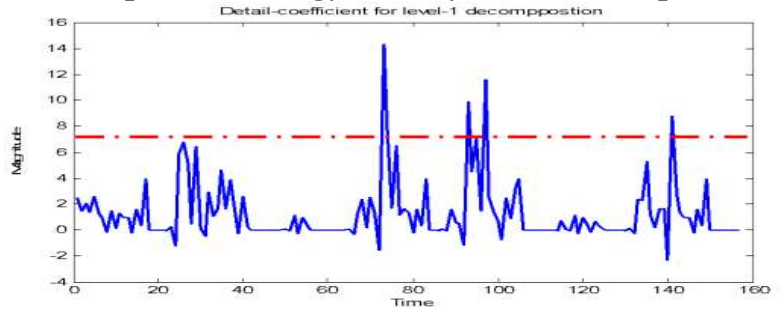

Figure 12: Extraction of Features from Selected Band -1

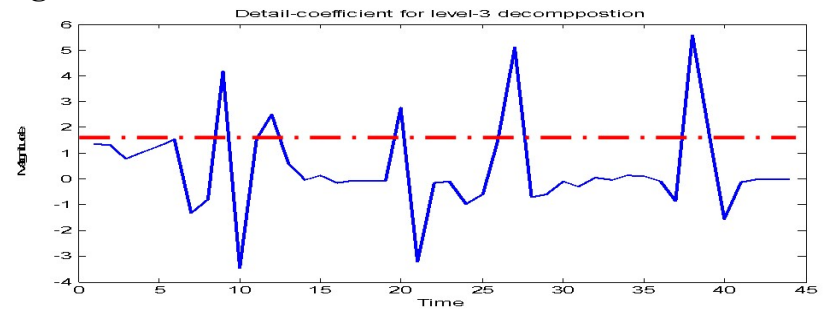

Figure 13: Extraction of Features from Selected Band -2 
The spectral energy density for each band is computed using power spectral density approach. Each band coefficients are averaged by the squared summation of its coefficients and energy is computed. From the band energy obtained, it is observed that, band 1 and 3 has comparatively higher energy density than the other two bands, so more good quality information is presented in these two bands. Using this power spectral density, desired bands for feature extraction is selected. Based on the energy derived, two highest energy density bands are selected, which are 1 and 3 in this case.

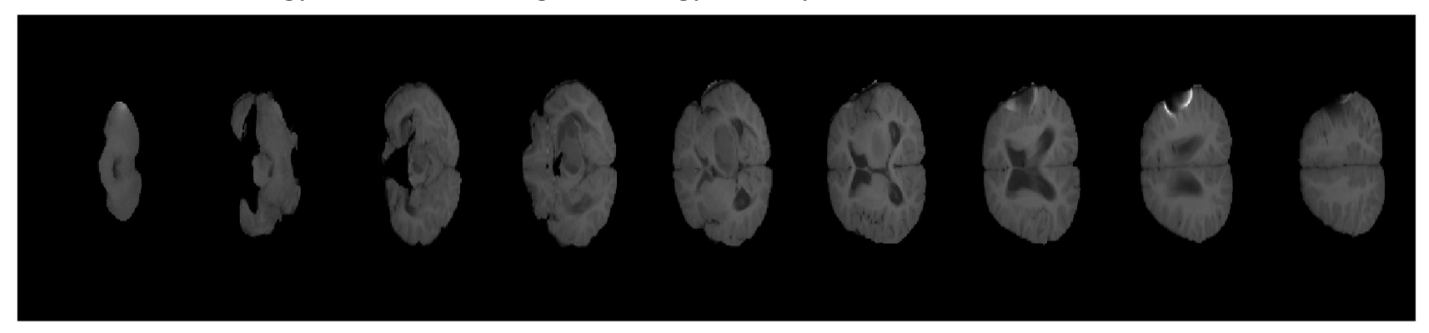

Figure 14: fMRI sample slices

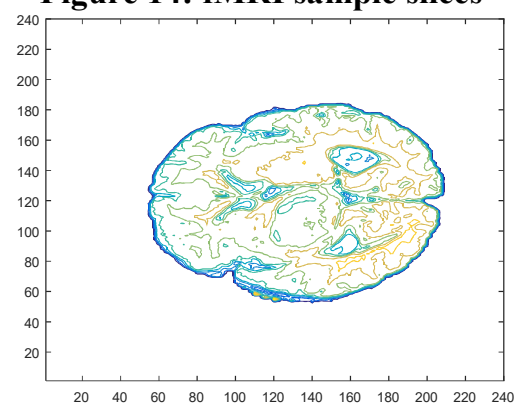

Figure 15: Spectral Plot for the MRI Sample

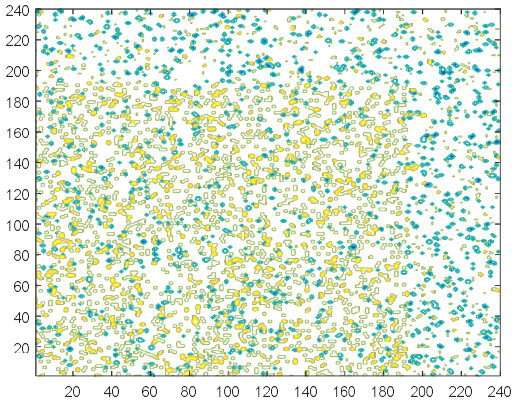

Figure 16: Correlative Spectral Map Plot

Figure 12 and 13 shows the two selected bands for feature extraction. The features are selected based on the similar procedure of maximum thresholding approach as used in conventional approach. For the two selected band, maximum peak values are found and a threshold of 0.6 of maximum peak is set as the threshold value. All the peaks falling above to this threshold is recorded as the feature magnitude with its corresponding coordinates, recording dominant coefficient peaks.

Active regions of MRI frame are analysed as shown in figure 16. These active regions are having higher spectral density. These high spectral density regions of fMRI are mapped with dominant coefficient peaks of EEG signal as shown in figure 16. Correlated features of EEG and fMRI are buffered in an array as a database. These features are then given to classifier to train the classifier.

This paper has adopted three methodologies to investigate the fairness in EEG-fMRI analysis and the prediction accuracy. First SCA approach [7], DCM approach [23] and DeepID approach [24]. Three methodologies are developed as an integrated model of EEG-fMRI, where the EEG signal is processed for mapping the voxel region movement based on the brain activity and recorded EEG peaks. The DCM approach computes the functional voxel by the Eigen space projection of prior and posterior temporal under the tumour affected regions. The DeepID approach is defined as a Neuro modelling of EEG-fMRI analysis using neural network modelling.

These approaches compute the spike detection based on a generalized linear modelling (GLM).

The comparative analysis made between the proposed IFA and the conventional methods are summarized in table 1 below. Table 1 represents the statistical report of SCA [7], DCM [23] and DeepID [24] and IFA for four subjects. The 
performance of EEG-fMRI analysis by the IFA has been demonstrated by comparing its recognition accuracy with SCA, DCM and DeepID.

In all the statistical cases, IFA has got the first rank, whereas the SCA is in the second position. The statistical report on the performance has ensured the superiority and the reliability of the proposed methodology.

Table 1: Evaluation Metric for the Developed Approach

\begin{tabular}{|c|c|c|c|c|c|c|c|c|}
\hline & Methodologies & $\begin{array}{c}\text { Accuracy } \\
(\%)\end{array}$ & Sensitivity & Specificity & Recall & Precision & \begin{tabular}{|c|c|} 
F- \\
Measure
\end{tabular} & CT(s) \\
\hline \multirow{4}{*}{$\begin{array}{c}\text { Subject } \\
1\end{array}$} & SCA[7] & 67.242 & 0.456 & 0.657 & 0.345 & 0.679 & 0.523 & 0.348 \\
\hline & IFA & 87.321 & 0.775 & 0.897 & 0.567 & 0.832 & 0.571 & 0.138 \\
\hline & DCM [23] & 64.322 & 0.545 & 0.685 & 0.332 & 0.643 & 0.502 & 0.365 \\
\hline & DeepID [24 ] & 62.221 & 0.599 & 0.612 & 0.314 & 0.631 & 0.471 & 0.377 \\
\hline \multirow{4}{*}{$\begin{array}{c}\text { Subject } \\
2\end{array}$} & SCA[7] & 79.281 & 0.588 & 0.665 & 0.458 & 0.666 & 0.621 & 0.443 \\
\hline & IFA & 89.500 & 0.789 & 0.854 & 0.524 & 0.820 & 0.652 & 0.337 \\
\hline & DCM [23] & 65.18 & 0.474 & 0.622 & 0.315 & 0.632 & 0.611 & 0.438 \\
\hline & DeepID[24] & 61.376 & 0.456 & 0.676 & 0.444 & 0.620 & 0.602 & 0.634 \\
\hline \multirow{4}{*}{$\begin{array}{c}\text { Subject } \\
3\end{array}$} & $\mathrm{SCA}[7]$ & 83.234 & 0.654 & 0.698 & 0.452 & 0.720 & 0.688 & 0.139 \\
\hline & IFA & 90.452 & 0.845 & 0.907 & 0.484 & 0.795 & 0.690 & 0.102 \\
\hline & DCM [23] & 75.323 & 0.616 & 0.692 & 0.355 & 0.720 & 0.614 & 0.241 \\
\hline & DeepID[24] & 71.234 & 0.601 & 0.656 & 0.332 & 0.715 & 0.610 & 0.278 \\
\hline \multirow{4}{*}{$\begin{array}{c}\text { Subject } \\
4\end{array}$} & $\mathrm{SCA}[7]$ & 85.420 & 0.678 & 0.623 & 0.558 & 0.723 & 0.600 & 0.231 \\
\hline & IFA & 92.820 & 0.772 & 0.865 & 0.582 & 0.810 & 0.680 & 0.189 \\
\hline & DCM [23] & 79.136 & 0.596 & 0.611 & 0.315 & 0.711 & 0.587 & 0.211 \\
\hline & DeepID[24 ] & 77.232 & 0.564 & 0.604 & 0.431 & 0.707 & 0.545 & 0.262 \\
\hline
\end{tabular}

\section{Conclusion}

This paper presents a new approach, feature mapping of EEG-fMRI analysis using integrated spectral correlation of time series mapping. Here empirical mode decomposition (EMD) has been introduced to extract frequency components of EEG signal. The fMRI slices and the EEG signals are correlated using the spectral correlation of selective spectral bands and the MRI spectral density in EEG-fMRI sample. The test validation illustrates the significance of the developed approach for different test analysis taking into consideration of artefacts present in EEG signal. The proposed approach has a significant minimization of feature counts and spectral mapping overhead by the appropriate spectral correlation decision. The performance of IFA, which has been referred as the proposed methodology, for the prediction of epileptic disorder is higher than the conventional models.

The proposed convergence mapping leads to proper decision of the selective region for epileptic diagnosis.

\section{References}

[1] Acharya, U. R., Molinari, F., Sree, S. V., Chattopadhyay, S., Ng, K. H., \& Suri, J. S. (2012). Automated diagnosis of epileptic EEG using entropies. Biomedical Signal Processing and Control, 7(4), 401-408.

[2] Adeli, E., Wu, G., Saghafi, B., An, L., Shi, F., \& Shen, D. (2017). Kernel-based joint feature selection and maxmargin classification for early diagnosis of Parkinson's disease. Scientific reports, 7, 41069.

[3] Al-Fahoum, A. S., \& Al-Fraihat, A. A. (2014). Methods of EEG signal features extraction using linear analysis in frequency and time-frequency domains. International Scholarly Research Notices, 2014.

[4] Alomari, M. H., Awada, E. A., Samaha, A., \& Alkamha, K. (2014). Wavelet-based feature extraction for the analysis of EEG signals associated with imagined fists and feet movements. Computer and Information Science, 7(2), 17.

[5] Alotaiby, T., Abd El-Samie, F. E., Alshebeili, S. A., \& Ahmad, I. (2015). A review of channel selection algorithms for EEG signal processing. EURASIP Journal on Advances in Signal Processing, 2015(1), 66.

[6] Álvarez-Meza, A. M., Velásquez-Martínez, L. F., \& Castellanos-Dominguez, G. (2015). Time-series discrimination using feature relevance analysis in motor imagery classification. Neurocomputing, 151, 122-129.

[7] EpifanioBagarinao, Satoshi Maesawa, Yuji Ito, NaotakaUsui, Jun Natsume, Hirohisa Watanabe, Minoru Hoshiyama, Toshihiko Wakabayashi, Gen Sobue, Shinji Naganawa, HaruoIsoda, (2018) Detecting sub-second changes in brain activation patterns during interictal epileptic spike using simultaneous EEG-fMRI. Clinical Neurophysiology, Vol.129, pp-377-389. 
[8] Birjandtalab, Javad, et al. (2017) Automated seizure detection using limited-channel EEG and non-linear dimension reduction. Computers in biology and medicine 82 49-58.

[9] Pachori, Ram Bilas, and Varun Bajaj. (2011) Analysis of normal and epileptic seizure EEG signals using empirical mode decomposition. Computer methods and programs in biomedicine 104.3: 373-381.

[10] Chella, F., Pizzella, V., Zappasodi, F., and Marzetti, L. (2016). Impact of the reference choice on scalp EEG connectivity estimation. J. Neural Eng. 13:036016.

[11] Duun-Henriksen, Jonas, et al. (2012) Channel selection for automatic seizure detection. Clinical Neurophysiology $123.1 \quad 84-92$.

[12] Vulliemoz S, Thornton R, Rodionov R, Carmichael DW, Guye M, Lhatoo S, McEvoy AW, Spinelli L, Michel CM, Duncan JS, Lemieux L. (2009). The spatio-temporal mapping of epileptic networks: combination of EEGfMRI and EEG source imaging. Neuroimage. Jul 1; 46(3):834-843.

[13] Brockmeier, A. J., Choi, J. S., Kriminger, E. G., Francis, J. T., \& Principe, J. C. (2014). Neural decoding with kernel-based metric learning. Neural computation, 26(6), 1080-1107.

[14] Brockmeier, A. J., Sanchez Giraldo, L. G., Emigh, M. S., Bae, J., Choi, J. S., Francis, J. T., et al. (2013).Information-theoretic metric learning: 2-D linear projections of neural data for visualization in Engineering in Medicine and Biology Society (EMBC), 2013 35th Annual International Conference of the IEEE (Osaka: IEEE), 5586-5589.

[15] Lopes, R., et al. (2012). Detection of epileptic activity in fMRI without recording the EEG. Neuroimage 60.3 1867-1879.

[16] Chang, Nai-Fu, et al. (2012). Channel selection for epilepsy seizure prediction method based on machine learning. 2012 Annual International Conference of the IEEE Engineering in Medicine and Biology Society. IEEE,

[17] Chen, S., Luo, Z., and Gan, H. (2016). An entropy fusion method for feature extraction of EEG. Neural Comput. Appl. 1, 1-7.

[18] Chu, C., Ni, Y., Tan, G., Saunders, C. J., and Ashburner, J. (2011). Kernel regression for fMRI pattern prediction. NeuroImage 56, 662-673.

[19] Cortes, C., Mohri, M., and Rostamizadeh, A. (2012). "Algorithms for learning kernels based on centered alignment." J.Mach. Learn. Res. 13, 795-828.

[20] Liu, Huan, and Lei Yu. (2005). Toward integrating feature selection algorithms for classification and clustering. IEEE Transactions on knowledge and data engineering 17.4 491-502.

[21] Daza-Santacoloma, G., Arias-Londo'no, J.D., Godino-Llorente, J. I., Sáenz-Lechón, N., Osma-Ruiz, V., and Castellanos-Dominguez, G. (2009). Dynamic feature extraction: an application to voice pathology detection. Intell. Autom. Soft Comput. 15, 667-682.

[22] Tomer Gazit, (2015) .fMRI-EEG database of epileptic seizure. ACTIVE project at Tel-Aviv Sourasky Medical Center (TASMC) Israel.

[23] Teresa Murta, Alberto Leal, Marta I. Garrido, Patrícia Figueiredo, (2012), Dynamic Causal Modeling of epileptic seizure propagation pathways: A combined EEG-fMRI study. NeuroImage 62 pp-1634-1642.

[24] Yongfu Haoa, Hui Ming Khooa, Nicolas von Ellenriedera, Natalja Zazubovitsa, Jean Gotmana, (2018).DeepIED: An epileptic discharge detector for EEG-fMRI based on deep Learning. NeuroImage: Clinical 17, pp-962-975.

[25] Keller TA,Just MA, Stenger VA (2018).Reading Span and the time-course of cortical activation in sentencepicture verification. Annual convention of the Psychonomic Society,Orlando,FL..

[26] A.N.Paithane and D.S.Bormane, (2015).Electrocardiogram signal analysis using empirical mode decomposition and Hilbert Spectrum. International conference on Pervasive Computing (ICPC), Pune, pp.1-4.

[27] Wang, Xiashuang, Guanghong Gong, and Ni Li. (2018).Multimodal fusion of EEG and fMRI for epilepsy detection. International Journal of Modeling, Simulation, and Scientific Computing $9.02: 1850010$.

[28] Omidvarnia, Amir, et al. (2019).Towards fast and reliable simultaneous EEG-fMRI analysis of epilepsy with automatic spike detection. Clinical Neurophysiology 130.3: 368-378.

[29] Abreu, Rodolfo, Alberto Leal, and Patrícia Figueiredo. (2019). Identification of epileptic brain states by dynamic functional connectivity analysis of simultaneous EEG-fMRI a dictionary learning approach. Scientific reports 9.1: 1-18.

[30] Paithane, A. N., Bormane, D. S., \& Patil, U. G. (2016). Novel algorithm for feature extraction and feature selection from electrocardiogram signal. International Journal of Computer Applications, 134(9), 6-9.

[31] S.V. Raut, D.M.Yadav, (2016). A Review on fMRI Signal Analysis and Brain Mapping Methodologies. AISC series of Springer International Conference on Computational Intelligence and Informatics-2016(ICCII-2016).

[32] Raut, S. V., \& Yadav, D. M. (2018). A decomposition model and voxel selection framework for fMRI analysis to predict neural response of visual stimuli. Biomedical Engineering/Biomedizinische Technik, 63(2), 163-175. 